\title{
Neuroprotection for Retinal Ganglion Cells
}

\author{
Muhammad Shahid* \\ Department of Pharmacy, Sarhad University of Science and Information Technology, Peshawar, Pakistan
}

\begin{abstract}
Retinal ganglion cells (RGCs) are important components of the visual pathway and are responsible for conveying retinal information through their axons, passing through the lateral geniculate nucleus and projecting into the visual cortex, thereby cognizing about the visual world. RGCs are energetically expensive dynamic cells that are highly susceptible to the same neuropathological mechanisms shared among other neurodegenerative conditions. These include deficits in mitochondrial subtleties, defects in axonal transport, and redox imbalance, eventually culminating in apoptosis and necrosis and resulting in RGC death. ${ }^{1}$ Degeneration of RGCs has significant impact on vision and is the leading cause of irreversible blindness. RGC degeneration is a prominent etiology underlying ophthalmic complications in patients with glaucoma,
\end{abstract} optic neuropathies and demyelinating disease.

Recently, the tetracycline antibiotic, minocycline has been investigated for potential neuroprotective effect on microglia activation-related degeneration of RGCs in both retinas using the unilateral optic nerve crush mouse model. ${ }^{2}$ It was demonstrated that minocycline can protect RGCs from secondary degeneration in the contralateral eye, while not influencing the primary RGC degeneration in the injured eye. Also, minocycline was shown to inhibit microglial activation, but the effect occurred considerably in the outer plexiform layer in both the ipsilateral and contralateral eyes following the crush injury. In the minocycline-treated animals, there was decreased microglial cell body area and circumference in the inner and outer plexiform layers in both eyes, while an increased number of nodes as well as length of branches in the contralateral inner plexiform layer was observed. It is noteworthy that all these characteristics were attended by a significant difference in the a- and b-wave amplitudes of electroretinogram after minocycline treatment, greatly improving the function of photoreceptor cells and bipolar cells in the contralateral eye. It was reasonably suggested, therefore, that minocycline partially inhibited the microglia activation and preserved the function of retinas. Minocycline has been designated as a neuroprotective agent, having an inhibitory effect on contralateral eye microglia activation. This has been corroborated by an another study, in which minocycline rescued RGCs in the contralateral retina but produced no effect on the injured retina. ${ }^{3}$ Although, in contrast, more authors have acknowledged that minocycline has a neuroprotective proclivity in the injured eye., ${ }^{4,5}$ Nevertheless, minocycline did disclose its potential in protecting the retina against secondary degeneration.

\section{Abbreviations: RGCs, retinal ganglion cells}

Received: August 10, 2020; Revised: August 26, 2020; Accepted: August 28, 2020

*Correspondence to: Muhammad Shahid, Department of Pharmacy, Sarhad University of Science and Information Technology, Peshawar 25000, Khyber Pakhtunkhwa, Pakistan. Tel: +92-3329283103, +92-91-5230931-32-33; Fax: +92-91-5230930; Email: shahidsalim_2002@hotmail.com; shahid.fls@suit.edu.pk

How to cite this article: Shahid M. Neuroprotection for Retinal Ganglion Cells. J Explor Res Pharmacol 2020;5(4):47-48. doi: 10.14218/JERP.2020.00023.
Consequently, minocycline can be a suitable conjunctive therapy choice in unilateral retinal diseases or traumatic brain injury conditions affected with optic nerve injury.

A neuroprotective agent can be beneficiary if it antagonizes the cytotoxic processes triggered by the neurotoxicological insult, along with substantiating the endogenous neuronal protective system. ${ }^{6}$ The current neuroprotective strategies are ineffective and produce only partial results, as these are focused on single pathways in neurodegeneration, thus necessitating a revisiting of designing such neuroprotective modalities that possess multitarget impacting propensity in the neuronal cell death cascades. In consequence, a neuroprotective agent may prove to be useful in improving survival and function of the non-regenerative RGCs against intracellular and environmental stresses. In view of this, a clinical neuroprotective strategy was developed in addition to lowering of intraocular pressure in patients with glaucoma. ${ }^{7}$ Consequently, a reliable neuroprotective agent for attenuating $\mathrm{RGC}$ degeneration is exceptionally timely.

A neuroprotection approach can be applied to restore vision in patients due to RGC damage or as preventive approach in individuals having a high risk of developing RGC degeneration. Preclinical animal models have proved useful in sorting out potential neuroprotective agents versus retinal neurodegeneration-like conditions. These approaches have even been shown to be valuable in detecting potential activity of compounds having neuroprotective affinity for RGCs and their beneficial proclivity has led to the prediction of clinical effectiveness. These include brimonidine, citicoline, triamcinolone acetonide, erythropoietin, prednisolone, methylprednisolone, idebenone, anti-vascular endothelial growth factor antibodies (bevacizumab, ranibizumab), and cyclosporine. ${ }^{8}$ An important aspect of RGC neuroprotection is the involvement of neurotrophic family of growth factors, including the neurotrophin family, the glial cell-line derived neurotrophic factor and ciliary neurotrophic factor in the neuronal regulation, survival, development and differentiation. ${ }^{9}$ The efficiency of these endogenous neuroprotective systems in preventing $\mathrm{RGC}$ degeneration is crucial ${ }^{10}$ and these tend to have value as the main mechanistic targets of potential approaches and technologies encompassing RGC neuroprotection.

The important findings underlying the degeneration of RGCs comes from the use of appropriate animal models, including heredity models $(\mathrm{C} 57 \mathrm{BL} / 6 \mathrm{~J} \times \mathrm{Krd} /+)$, transgenic models $\left(\mathrm{Bax}^{-/-}\right.$, $\mathrm{C} 3 \mathrm{H} \times \mathrm{C} 57 \mathrm{BL} / 6)$, and inducible models (N-methyl-D-aspartate [commonly referred to as NMDA], experimental autoimmune encephalomyelitis, optic nerve crush injury, transient ischemia, and light-induced retinal cell degeneration). ${ }^{11,12}$ Although these models have made a major contribution to the field of ophthalmology and vision research, the progress in this area of research has been mired by innate translational problems. These include the rarity of human pathological tissues, accurate modelling of complex multifactorial disease state, variability in disease phenotypic expression, 
differences in outcome measures, inclination in the time-course of retinal degenerative changes in animals, apparent differences in ocular anatomy and physiology of tear flow and mixing compared to humans, the greater surface-to-volume ratio, increased basal metabolic rate and polyunsaturated nature of cellular membranes of small animals. Canine studies have shown some promise in improving the predictive power of preclinical ophthalmic research; nonetheless, they are presented with inherent limitations, including mixed genetic background affecting the variability in disease characteristics, in addition to associated economic and ethical challenges. ${ }^{13}$ It is worthwhile to bear in mind that these are important matters in extrapolating experimental data from bench to clinic. This may explain why many effective neuroprotective agents in preclinical research fail in clinical trials.

In the clinic, a true parameter of RGC neurodegeneration is currently a challenge, as surrogate measures are utilized to estimate the integrity of RGCs. ${ }^{14}$ In this respect, translation of potential RGC neuroprotective strategies into clinical ophthalmic practice remains an issue. Another confounding factor is that in animal studies, neuroprotective agents are administered prior to the RGC damage. This limits their translational relevance when relating any beneficial effect to therapeutic intervention in patients who are already diagnosed with retinal disease. ${ }^{15}$ It should also be kept in mind that RGCs exhibit tremendous diversity and RGC types appear to differ profoundly in their ability to survive insults. ${ }^{16}$ In the case of minocycline, oral treatment appeared to have potential efficacy in increasing visual acuity and reducing macular edema in patients with diabetes mellitus. ${ }^{17}$ Ostensibly, minocycline may well have a neuroprotective value; though in consideration of the foregoing discussion and the failure to achieve any convincible therapeutic benefits with other neuroprotective agents, uncertainty prevails about minocycline's clinical efficacy in RGC degenerative conditions. Additionally, there are cases of minocycline-induced intracranial hypertension and papilledema; ${ }^{18,19}$ these markedly confound the prospective efficacy of minocycline in patients with retinal degeneration. This emphasizes the duality of challenges coupled to preclinical investigation of neuroprotective agents and their therapeutic effectiveness; so, clinically, combinatorial approaches should be considered to maximize the neuroprotection of RGCs.

\section{Acknowledgments}

This was an invited commentary on: Rui Guo. Minocycline protects against the degeneration of retinal neurons in mice. Journal of Exploratory Research in Pharmacology, 2020. doi: 10.14218/ JERP.2020.00015.

\section{Funding}

None.

\section{Conflict of interest}

The author has no conflicts of interest to declare.

\section{References}

[1] Goldberg JL, Corredor RG. Retinal Ganglion Cell Life and Death Mechanisms and Implications for Ophthalmology. Eur Ophthalmic Rev 2009;3(2):109-112. doi:10.17925/EOR.2009.03.02.109.

[2] Guo R. Minocycline Protects Against the Degeneration of Retinal Neurons in Mice. J Explor Res Pharmacol 2020. doi:10.14218/ JERP.2020.00015.

[3] Lucas-Ruiz F, Galindo-Romero C, Rodríguez-Ramírez KT, Vidal-Sanz M, Agudo-Barriuso $\mathrm{M}$. Neuronal death in the contralateral un-injured retina after unilateral axotomy: role of microglial cells. Int J Mol Sci 2019;20(22):5733. doi:10.3390/ijms20225733.

[4] Jiao X, Peng Y, Yang L. Minocycline protects retinal ganglion cells after optic nerve crush injury in mice by delaying autophagy and upregulating nuclear factor-KB2. Chin Med J (Engl) 2014;127(9):1749-1754.

[5] Levkovitch-Verbin H, Kalev-Landoy M, Habot-Wilner Z, Melamed S. Minocycline delays death of retinal ganglion cells in experimental glaucoma and after optic nerve transection. Arch Ophthalmol 2006;124(4):520-526. doi:10.1001/archopht.124.4.520.

[6] Carletti B, Fpiemonte F, Rossi F. Neuroprotection: the emerging concept of restorative neural stem cell biology for the treatment of neurodegenerative diseases. Curr Neuropharmacol 2011;9(2):313-317. doi:10.2174/157015911795596603.

[7] Levin LA. Retinal ganglion cells and neuroprotection for glaucoma. Surv Ophthalmol 2003;48(2):S21-S24. doi:10.1016/s00396257(03)00007-9.

[8] Boia R, Ruzafa N, Aires ID, Pereiro X, Ambrósio AF, Vecino E, et al. Neuroprotective strategies for retinal ganglion cell degeneration: current status and challenges ahead. Int J Mol Sci 2020;21(7):2262. doi:10.3390/ijms21072262.

[9] Hennigan A, O'callaghan R, Kelly A. Neurotrophins and their receptors: roles in plasticity, neurodegeneration and neuroprotection. Biochem Soc Trans 2007;35(2):424-427. doi:10.1042/BST0350424.

[10] Pietrucha-Dutczak M, Amadio M, Govoni S, Lewin-Kowalik J, Smedowski A. The role of endogenous neuroprotective mechanisms in the prevention of retinal ganglion cells degeneration. Front Neurosci 2018;12:834. doi:10.3389/fnins.2018.00834.

[11] Hafezi F, Grimm C, Simmen BC, Wenzel A, Remé CE. Molecular ophthalmology: an update on animal models for retinal degenerations and dystrophies. Br J Ophthalmol 2000;84(8):922-927. doi:10.1136/ bjo.84.8.922.

[12] Niwa M, Aoki H, Hirata A, Tomita H, Green PG, Hara A. Retinal cell degeneration in animal models. Int J Mol Sci 2016;17(1):110. doi:10.3390/ijms17010110.

[13] Sebbag L, Mochel JP. An Eye on the Dog as the Scientist's Best Friend for Translational Research in Ophthalmology: Focus on the Ocular Surface. Med Res Rev 2020. doi:10.1002/med.21716.

[14] Smith CA, Vianna JR, Chauhan BC. Assessing retinal ganglion cell damage. Eye (Lond) 2017;31(2):209-217. doi:10.1038/eye.2016.295.

[15] Khatib TZ, Martin KR. Protecting retinal ganglion cells. Eye (Lond) 2017;31(2):218-224. doi:10.1038/eye.2016.299.

[16] Sanes JR, Masland RH. The types of retinal ganglion cells: current status and implications for neuronal classification. Annu Rev Neurosci 2015;38:221-246. doi:10.1146/annurev-neuro-071714-034120.

[17] Cukras CA, Petrou P, Chew EY, Meyerle CB, Wong WT. Oral minocycline for the treatment of diabetic macular edema (DME): results of a phase I/II clinical study. Invest Ophthalmol Vis Sci 2012;53(7):38653874. doi:10.1167/iovs.11-9413.

[18] Fraser CL, Biousse V, Newman NJ. Minocycline-induced fulminant intracranial hypertension. Arch Neurol 2012;69(8):1067-1070. doi:10.1001/archneurol.2012.144.

[19] Chiu AM, Chuenkongkaew WL, Cornblath WT, Trobe JD, Digre KB, Do$\tan \mathrm{SA}$, et al. Minocycline treatment and pseudotumor cerebri syndrome. Am J Ophthalmol 1998;126(1):116-121. doi:10.1016/s0002 9394(98)00063-4. 